\title{
Leveraging Deep Learning for Visual Odometry Using Optical Flow
}

\author{
Tejas Pandey*, Dexmont Pena, Jonathan Byrne (D) and David Moloney \\ Intel Research \& Development, W23 CX68 Leixlip, Ireland; dexmont.pena@intel.com (D.P.); \\ jonathan.byrne@intel.com (J.B.); david.moloney@intel.com (D.M.) \\ * Correspondence: tejas.pandey@intel.com
}

check for updates

Citation: Pandey, T.; Pena, D.; Byrne, J.; Moloney, D. Leveraging Deep Learning for Visual Odometry Using Optical Flow. Sensors 2021, 21, 1313. https://doi.org/10.3390/s21041313

Academic Editor: Steve Vanlanduit Received: 8 January 2021

Accepted: 9 February 2021

Published: 12 February 2021

Publisher's Note: MDPI stays neutral with regard to jurisdictional clai$\mathrm{ms}$ in published maps and institutional affiliations.

Copyright: () 2021 by the authors. Licensee MDPI, Basel, Switzerland. This article is an open access article distributed under the terms and conditions of the Creative Commons Attribution (CC BY) license (https:// creativecommons.org/licenses/by/ $4.0 /)$.

\begin{abstract}
In this paper, we study deep learning approaches for monocular visual odometry (VO). Deep learning solutions have shown to be effective in VO applications, replacing the need for highly engineered steps, such as feature extraction and outlier rejection in a traditional pipeline. We propose a new architecture combining ego-motion estimation and sequence-based learning using deep neural networks. We estimate camera motion from optical flow using Convolutional Neural Networks (CNNs) and model the motion dynamics using Recurrent Neural Networks (RNNs). The network outputs the relative 6-DOF camera poses for a sequence, and implicitly learns the absolute scale without the need for camera intrinsics. The entire trajectory is then integrated without any postcalibration. We evaluate the proposed method on the KITTI dataset and compare it with traditional and other deep learning approaches in the literature.
\end{abstract}

Keywords: visual odometry; ego-motion estimation; deep learning

\section{Introduction}

Visual odometry [1] is the challenging task of camera pose estimation and robot localization based only on visual feedback. It represents one of the fundamental problems of computer vision, and forms an integral part of Simultaneous Localization And Mapping (SLAM) and robot kinematics. It is a long-standing challenge in the field, and is being worked on for several decades [2,3].

Visual odometry forms an integral component for autonomous agents. A self-driving agent is responsible for being aware of the environment and other moving objects in the scene, as well as its own relative movements. Given a sequence of frames, the agent needs to keep track of the relative inter-frame motion, as well as positioning on a global scale. Marginal errors called "drift" are introduced in the relative motion prediction. They accumulate over the length of a path and propagate throughout the global trajectory, leading to significant errors.

Classical visual odometry is dominated by stereoscopic and RGB-D cameras where information such as scale, which gets lost during projection mapping, can be recovered to reduce the drift [4-7]. Others rely on sensor fusion, where data from multiple sensors, such as LIDAR, IMU, and GPS are fused to come up with more reliable and robust solutions $[8,9]$. Recently, there has been an increased interest in problems regarding ego-motion with the rise of autonomous agents navigating in unfamiliar dynamic environments, such as selfdriving cars and UAV drones, leading to a demand for cheap and reliable commodity solutions. Integrating multiple cameras with different sensors requires not only space and expensive hardware, but also sufficient processing power and energy. Even stereoscopic cameras are constrained if the robot is too small and cameras are too close. Monocular visual odometry has the advantage of using only a single camera; however, it is significantly more challenging due to the number of ambiguities involved. These challenges include loss of scale, dynamic environments, inconsistent changes in illumination, and occlusion [10]. Traditional approaches can be divided into two categories: direct, and feature-based. 
Feature-based methods [11-13] extract geometrically consistent features [14] and track them across subsequent frames. The camera motion is estimated by minimizing reprojection errors between feature pairs. Though feature-based methods are robust to noise, they fail to work in sparse, textureless environments where the algorithm is unable to generate suitable features.

Direct methods [15] track the intensity of pixels and estimate camera motion by minimizing photometric errors. They are more robust in environments lacking visual features, but are susceptible to sudden changes between subsequent frames.

In computer vision, deep learning-based approaches have outperformed traditional methods in tasks such as object classification and detection [16-18]. However, these approaches are unsuitable for geometric tasks, as they were designed to be translation/orientation invariant, rather than equivariant. They lose track of spatial relationships and ignore physical features such as size, velocity, and orientation. Different adaptions were designed to overcome this problem $[19,20]$.

In this paper, we first study the problem of visual odometry using deep neural networks. We search for deep learning approaches to overcome the limitations present in direct and feature-based methods. Deep neural networks learn feature representations in higher dimensions and can replace the engineering effort required in integrating and fine-tuning individual components [1,21].

We then propose a pipeline using CNNs that takes optical flow as input and directly estimates a relative 6-DOF camera pose. We augment the network with RNNs, as represented in Figure 1, to implicitly model relationships between subsequent frames in higher dimensions.

The rest of the paper goes as follows-Section 2 reviews related work and Section 3 comprises our proposed model, followed by experimental results in Section 4 and the conclusion in Section 5.

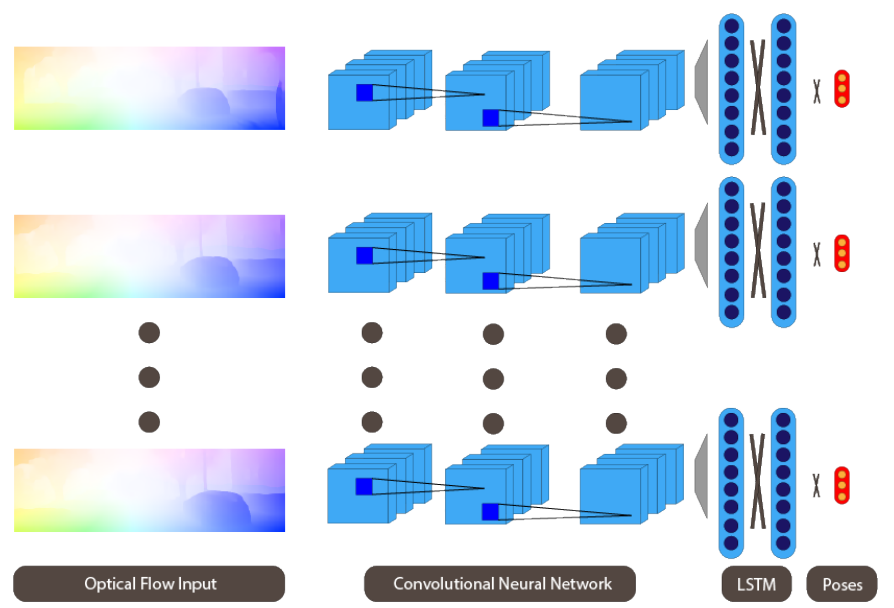

Figure 1. Overview of our approach: Optical flow is passed on to CNNs, extracting relevant features, which are in turn passed on to the RNNs.

\section{Related Work}

Monocular visual odometry is the task of tracking the flow of information from a single camera and predicting the path undertaken by it. Traditional methods $[12,22,23]$ are solely insufficient and have to rely on external parameters to account for the ambiguities involved, such as scale, whereas deep learning approaches [24,25] are self-sufficient and show improved results compared to older traditional methods.

Recent deep learning approaches [26,27] propose taking a pre-trained FlownetS [28] and re-purposing it to predict relative poses. The FlownetS architecture, given two RGB images, uses a single network for feature extraction and matching to estimate optical flow. The hypothesis is that the features learned for capturing the relationship between frames 
for estimating optical flow should also be suitable for the task of predicting the relative pose between them.

Another approach is to directly use optical flow as input [26,29] and regress the relative pose based on learned priors. The advantage here is that it also allows us to decouple the problem of visual odomtery into feature extraction and motion estimation.

\subsection{Sequence-Based Modelling}

Wang et al. [24] were the first ones to propose a sequence-based model. They argued that CNNs were not sufficient to model the visual odometry pipeline (VO). While CNNs are useful for learning visual features, they cannot capture motion dynamics. They proposed using LSTMs to capture the temporal relationship between frames. However, LSTMs are not suitable for working with high-dimensional data, such as high-resolution images. Thus, the FlownetS architecture is augmented with LSTMs to model the entire VO pipeline. Feature representations extracted from CNNs are passed onto LSTMs, which output pose estimates at every timestep.

Parisotto et al. [27] countered that while LSTMs can model relationships between frames, they cannot influence past outputs, and proposed an attention-based, neural globalbundle adjustment network. The output features from FlownetS are passed onto the global-adjustment network, which uses temporal convolutions to account for drift over long trajectories. Subsequently predicted trajectories can be refined by recursively passing the outputs back to the global-adjustment network.

Jiao et al. [25] proposed an iterative update to [24] using bi-directional LSTMs, and outperformed [24] on the KITTI dataset. Bi-directional LSTMs model relationships for both forward and backward sequences. LSTMs learn to model the conditional probability between poses. A bi-directional LSTM would open up the probable space by learning from future sequences as well.

\subsection{Optical Flow-Based Visual Odometry}

Costante and Ciarfuglia [30] argued that deep learning approaches involved not only learning visual cues, but camera intrinsics as well. They recommended decoupling the learning of visual and motion features for better generalization. Rather than use a single network for feature extraction and motion estimation, they proposed an auto-encoder to capture an embedding of the optical flow and thereby predict the relative pose based off of it. They formed a multi-objective loss function to minimize the error in reconstruction of optical flow and camera pose estimation, thus ensuring the captured embedding was suitable for motion estimation. They were able to outperform traditional monocular approaches $[13,31]$. However, a multi-objective function can hinder the performance of motion prediction where the network can struggle to balance the loss of optical flow reconstruction and pose estimation. Moreover, the two tasks may not share favourable features, thus reducing the accuracy of both reconstruction and pose prediction [32].

Costante et al. [29] used optical flow as an input to estimate the camera pose. In order to retain the spatial relationship, they proposed splitting the input optical flow into quadrants and passing individual quadrants into a combination of 5 different neural networks - one for every quadrant, and one for the entire optical flow. Different regions add value for specific directions, and thus, splitting the optical flow into quadrants allowed us to preserve such information from shared parameters. Outputs of all networks were concatenated in order to regress the relative translation and rotation parameters. They were able to outperform traditional monocular methods as well. However, their approach required five networks, which is computationally expensive and memory-intensive.

Muller and Savakis [26] proposed using the same architecture as FlownetS while passing 2D optical flow vectors as input, rather than a six-channel concatenated RGB pair. They used the same hyper-parameters due to FlownetS' success on RGB images and performed pose estimation using a single network, passing optical flow vectors as input. However, a 
network designed for learning relationships between RGB pairs may not be sufficient for the task of pose estimation from optical flow, as it was unable to outperform [29].

\subsection{Contributions}

Costante et al. [29] argued that CNNs were translation-invariant, and proposed splitting the optical flow into quadrants to retain motion information. However, their approach was computationally expensive. Thus, our proposed solution is more similar to [26], of conventional regression. However, the approach by Muller and Savakis [26] of adopting the same FlownetS architecture for the task of visual odometry, while promising, could not outperform Costante et al.

Deep learning networks, such as Mobilenet [16] and Resnet [17], could outperform traditional approaches for image classification, but not for optical flow estimation. Thus, Flownets [28] came into inception as networks more suitable for the task of optical flow estimation. Now, rather than using Flownets for the task of pose estimation as well, we propose an architecture optimized for estimating relative poses from optical flow.

Since the inception of Flownets, there has been an increased interest in augmentation with optical flow in deep learning. It has shown promising results with its introduction in applications such as frame interpolation for slow-motion videos [33] and superresolution [34]. However, optical flow inputs have underperformed in the field of visual odometry. Our work shows the benefits of optical flow for monocular visual odometry.

Wang et al. [24] argued CNNs were insufficient by themselves to model the visual odometry pipeline from raw RGB images, and proposed augmenting CNNs with LSTMs, which can implicitly learn to model the temporal relationship between subsequent frames. To exploit this advantage, we also augment our optical flow-based network with LSTMs to reduce the drift errors in a sequence.

\section{Visual Odometery Using Optical Flow}

In our approach, we combine optical flow-based ego-motion estimation with sequencebased modelling. We take in optical flow as input and use CNNs to learn a dense feature description, which is then passed onto bi-LSTMs. Our network models spatio-temporal relationships across a sequence to frame the entire visual odometry pipeline (Figure 1). We show our proposed architecture in Figure 2. However, calculating optical flow [35] has always been a computationally expensive task. Fischer et al. [28] proposed two novel architectures, FlownetS and FlownetC, for estimating the optical flow given two RGB images. While the FlownetS architecture used a single network for feature extraction and matching, FlownetC used a siamese network for feature extraction and a novel correlation layer for feature matching in higher dimensions. The networks were able to deliver competitive results with real-time frame rates. Flownet was followed by Flownet2 [36], which proposed stacking the different architectures together and significantly improved upon optical flow estimates. Since then, there has been an increased interest in leveraging deep learning for predicting optical flow.

For our proposed method, we use LiteFlowNet [37] to predict our optical flow input. LiteFlowNet was designed on the principles of pyramid feature scaling, feature warping, and cost volume. Rather than scaling images, feature pyramids are generated at different scales to make the network more robust to noise and changes in illumination. It then uses feature warping to generate a cost volume representation, and introduces a regularization layer to refine the optical flow estimate. LiteFlowNet is 30 times smaller than Flownet2, and outperformed PWC-Net [38] and Flownet2 on the KITTI flow 2012 benchmark [39]. We show a visualization of the generated optical flow in Figure 3. 


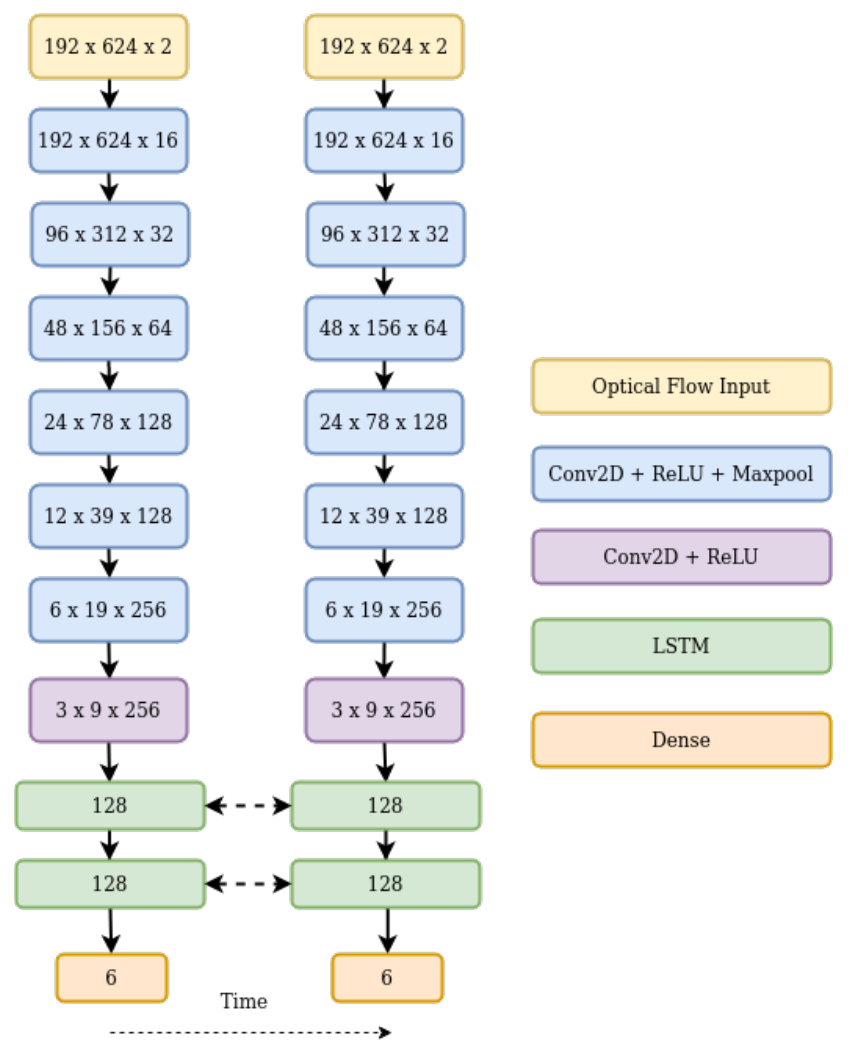

Figure 2. Proposed architecture.

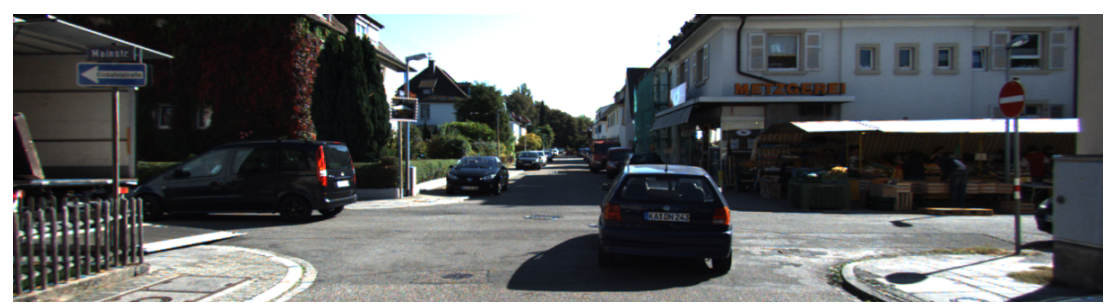

(a) Frame at time $t$.

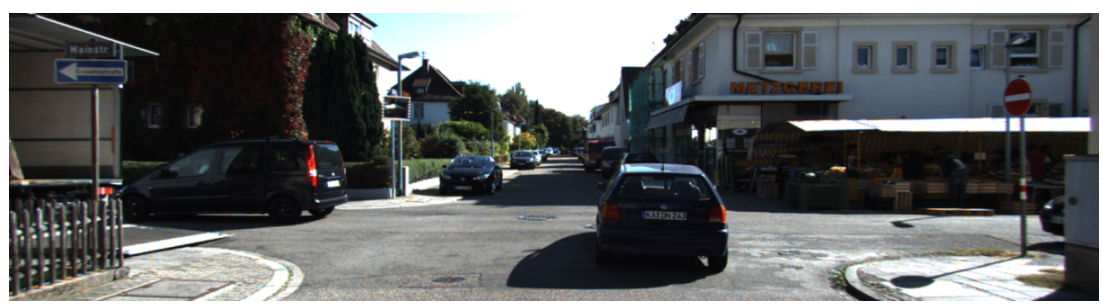

(b) Frame at time $t+1$.

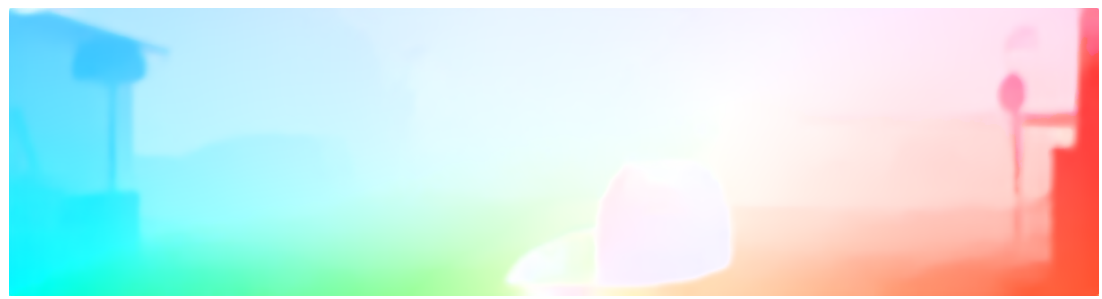

(c) Resulting optical flow.

Figure 3. Example frames from the KITTI dataset [40] and resulting optical flow using LiteFlowNet [37]. 


\subsection{Optical Flow}

The rise in popularity of deep learning for image classification came due to the availability of large-scale datasets. Ideally, we would want our dataset to represent the real world. However, there is a lack of comprehensive data for geometric tasks, such as visual odometry, which require recording and manually calibrating data from different sensors. To overcome this limitation, we chose to work with optical flow rather than RGB images, as optical flow is an encoded representation of flow of motion. It is scene-invariant and maps the pixel correspondences between subsequent frames. We believe an optical flow-based method would generalize better compared to RGB images.

\subsection{CNN Architecture}

Extracting features from optical flow is suitable for a $\mathrm{CNN}$, as it requires extracting patterns from encoded flow vectors. However, existing off-the-shelf feature extractors, such as Mobilenet and Resnet, were trained on Imagenet, which is of a different domain. Thus, we propose our own network for extracting features from optical flow. Rather than use the FlownetS architecture which was designed for RGB images, we did a parameter search for a network suitable for optical flow inputs, through hyper-parameter tuning [41]. The network consists of seven convolution layers with max pooling applied after every layer, excluding the last one. We apply a rectified linear unit as our activation function. The CNN architecture is described in Table 1. We use a small architecture to avoid the problem of over-fitting, as we are working on a relatively small dataset.

Table 1. Structure of CNN.

\begin{tabular}{cccc}
\hline Layer & Kernel Size & Strides & Channels \\
\hline Conv2D & 3 & 1 & 16 \\
MaxPool & & 2 & 32 \\
Conv2D & 3 & 1 & 64 \\
MaxPool & & 2 & 128 \\
\hline Conv2D & 3 & 1 & 128 \\
MaxPool & 3 & 2 & 256 \\
\hline Conv2D & 3 & 1 & \\
MaxPool & 3 & 1 & 256 \\
\hline Conv2D & 3 & 1 & \\
\hline MaxPool & 3 & 2 & \\
\hline Conv2D & 3 & 1 & \\
MaxPool & 3 & & \\
\hline Conv2D & & & \\
\hline
\end{tabular}

We pass 2D optical flow vectors as input, where every vector acts as a relative pose for that point in space. At this stage, our network has to implicitly estimate the relative pose for all points and accumulate the results to come up with a final estimate for camera motion.

\subsection{Sequence-Based Modelling}

Sequence-based modelling for visual odometry was first proposed by [24]. RNNs have shown remarkable performance on Natural Language Processing (NLP) tasks [42], and have been used extensively for sequence-based learning [43]. RNNs have the ability to learn relationships over time as they can access feature outputs of the previous cell.

Given feature $x_{t}$ at time $t$, RNN is updated at time $t$ by:

$$
\begin{array}{r}
h_{t}=\mu\left(W_{x h} x_{t}+W_{h h} h_{t-1}+b_{h}\right) \\
y_{t}=W_{h y} h_{t}+b_{y}
\end{array}
$$


where $h_{t}$ is the state variable of the hidden layer at time $t, y_{t}$ an output variable. $W_{h h}$ and $W_{x h}$ are the weight matrix of the hidden layers and input features, respectively, and $b_{h}$ and $b_{y}$ are the bias vectors of the hidden layers and input features, respectively. $\mu$ is a non-linear activation function, generally consisting of tanh and hard sigmoid.

However, RNNs suffer from vanishing gradients if the gradients pass through many timesteps. Thus, to account for the vanishing gradients, LSTMs were introduced with a three-gate system, namely, an input gate, output gate, and a forget gate. The gates determine how to update the current state. LSTMs avoid the problem of vanishing gradients, and can capture relationships over long periods. For our paper, we follow the approach proposed by [25] and use a bi-directional LSTM which consists of two LSTMs. as follows:

The equations for the input gate, forget gate, output gate, and input cell state are

$$
\begin{array}{r}
f_{t}=\sigma_{g}\left(W_{f} x_{t}+U_{f} h_{t-1}+b_{f}\right) \\
i_{t}=\sigma_{g}\left(W_{i} x_{t}+U_{i} h_{t-1}+b_{i}\right) \\
o_{t}=\sigma_{g}\left(W_{o} x_{t}+U_{o} h_{t-1}+b_{o}\right) \\
\widetilde{C}=\tanh \left(W_{C} x_{t}+U_{c} h_{t-1}+b_{c}\right)
\end{array}
$$

where $W_{f}, W_{i}, W_{o}$, and $W_{c}$ are the weight matrices for the forget gate, input gate, output gate, and input cell state, while $U_{f}, U_{i}, U_{o}$ and $U_{c}$ are the weight matrices connecting the previous cell output state to the three gates and the input cell state. $b_{f}, b_{i}, b_{o}$ and $b_{c}$ are the bias vectors for the forget gate, input gate, output gate, and input cell state. $\sigma_{g}$ is the gate activation function, a hard sigmoid function, and tanh is the hyperbolic tangent function. The cell output state $C_{t}$ and the hidden state $h_{t}$ are as follows:

$$
\begin{array}{r}
C_{t}=f_{t} * C_{t-1}+i_{t} * \widetilde{C}_{t} \\
h_{t}=o_{t} * \tanh \left(C_{t}\right) .
\end{array}
$$

Bi-directional LSTMs consists of two LSTMs. Thus, the output is represented as:

$$
y_{t}=\sigma\left(\overrightarrow{h_{t}}, \overleftarrow{h_{t}}\right)
$$

where $\sigma$ is the function used to combine the two output sequences and $y_{t}$ is the output variable at time $t$.

Bi-directional LSTMs learns from both forward-sequence $\vec{h}$ from time $t_{1} \ldots t_{n}$, as well as backward-sequence $\overleftarrow{h}$ from time $t_{n} \ldots t_{1}$.

The last layer of our CNN block passes a dense feature representation to the bi-LSTMs to model the temporal relationships between feature representations in higher dimensions.

We use a pair of bi-LSTMs as proposed by [24] with 128 units, thus a total of 256 units, and a timestep of five frames. The bi-LSTMs are then connected to a fully connected layer which estimates the 6-DOF pose.

\subsection{Loss Function}

We use the Mean Squared Error (MSE) loss to minimize the euclidean distance between the ground-truth pose and prediction:

$$
\underset{\theta}{\operatorname{argmin}} \frac{1}{N} \sum_{i=1}^{N} \sum_{k=1}^{K}\left\|\hat{p}_{k}^{(i)}-p_{k}^{(i)}\right\|_{2}^{2}-w\left\|\hat{q}_{k}^{(i)}-q_{k}^{(i)}\right\|_{2}^{2}
$$

where $p$ and $q$ denote translation and rotation parameters and $N$ and $K$ the number of samples and dimensions, respectively. 


\section{Results}

In this section, we go over our implementation and training details and evaluate our model on the KITTI dataset [40].

\subsection{Experimental Setup}

The network was trained using Tensorflow 2.0 with an Intel i7-6850K, Nvidia Geforce Titan X Maxwell and 32 GBs of RAM.

\subsection{Dataset}

The KITTI dataset [40] consists of 22 sequences. Only sequences $00-10$ have their ground truth provided. The rest are saved for testing and only supplied with raw sensor data. The dataset includes stereo images captured at $10 \mathrm{fps}$, as well as IMU and LIDAR. For our purposes, we only work with the left-most stereo camera. The dataset consists of a car driving in dense residential urban environments and sparse highways. To train our model, we divide the dataset into training and testing data. We use sequences $00,02,04$, 06,08 , and 10 for training our model and sequences $03,05,07$, and 09 for validation. We avoid working with sequence 01 since the car is driving at high speeds, resulting in large displacements between frames.

The ground-truth is comprised of $4 \times 4$ transformation matrices. We calculate the relative poses for each RGB pair and convert them to an euler representation comprised of three translation and rotation parameters each.

\subsection{Implementation}

We use the Adam optimizer with a learning rate of 0.001. Learning rate decay is employed to achieve faster convergence, and the network is trained for 100 epochs. We first train the network to infer relative poses from optical flow, and then augment it with bi-directional LSTMs.

\subsection{Results}

We evaluate our model on sequences $03,05,07$, and 09 to realize the generalization of our network. The evaluation is based on the KITTI odometry benchmark [39]. Translation and rotation errors are calculated for sub-sequences of lengths ranging from 100 to $800 \mathrm{~m}$ every $100 \mathrm{~m}$. Then, the averaged root-mean-squared error is considered. We compare our results vs. VISO2 [13] and MagicVO [25]. VISO2 is an open-source, feature-based solution for visual odometry. It minimizes re-projection errors between sparse feature matches and supports monocular and stereo-based configurations. We show comparisons against both configurations. MagicVO is a deep learning approach that augments FlownetS with bi-directional LSTMs. We chose to compare against MagicVO, as their results outperform other approaches in the literature, and thus they act as a good baseline. Since their implementation is not public, we compare against results published in their paper. We report our results in Table 2. We also draw a quantitative and qualitative comparison against VISO2. We show quantitative comparisons in Figure 4, and qualitative ones in Figure 5. 
Table 2. Comparison of proposed method vs. monocular and stereo-based VISO2 and MagicVO. • t: average translation drift $(\%)$ on length of $100-800 \mathrm{~m}$. $\bullet \mathrm{r}$ : average rotation $\operatorname{drift}\left({ }^{\circ} / 100 \mathrm{~m}\right)$ on length of $100-800 \mathrm{~m}$.

\begin{tabular}{ccccccccccc}
\hline Seq. & \multicolumn{2}{c}{ Proposed } & \multicolumn{2}{c}{ Proposed (LSTM) } & \multicolumn{2}{c}{ VISO2_M } & \multicolumn{2}{c}{ VISO2_S } & \multicolumn{2}{c}{ MagicVO } \\
\hline & $\mathrm{t}(\%)$ & $\mathrm{r}\left(^{\circ}\right)$ & $\mathrm{t}(\%)$ & $\mathrm{r}\left(^{\circ}\right)$ & $\mathrm{t}(\%)$ & $\mathrm{r}\left({ }^{\circ}\right)$ & $\mathrm{t}(\%)$ & $\mathrm{r}\left(\left(^{\circ}\right)\right.$ & $\mathrm{t}(\%)$ & $\mathrm{r}\left({ }^{\circ}\right)$ \\
\hline 03 & 4.85 & 2.54 & 9.82 & 3.64 & 10.57 & 1.73 & $\mathbf{2 . 9 4}$ & $\mathbf{1 . 0 9}$ & 4.95 & 2.44 \\
\hline 05 & 2.89 & 1.22 & 3.03 & 1.23 & 19.02 & 4.21 & 2.40 & $\mathbf{1 . 1 5}$ & $\mathbf{1 . 6 3}$ & 2.25 \\
\hline 07 & $\mathbf{2 . 5 6}$ & 2.15 & 6.43 & 3.39 & 34.16 & 9.98 & 2.67 & 1.61 & 2.61 & $\mathbf{1 . 0 8}$ \\
\hline 09 & $\mathbf{2 . 5 4}$ & $\mathbf{0 . 9 0}$ & 5.05 & 1.90 & 5.76 & 1.05 & 2.86 & 1.14 & 5.43 & 2.27 \\
\hline Avg. & 3.21 & 1.70 & 6.08 & 2.54 & 17.38 & 4.24 & $\mathbf{2 . 7 2}$ & $\mathbf{1 . 2 5}$ & 3.66 & 2.01 \\
\hline
\end{tabular}

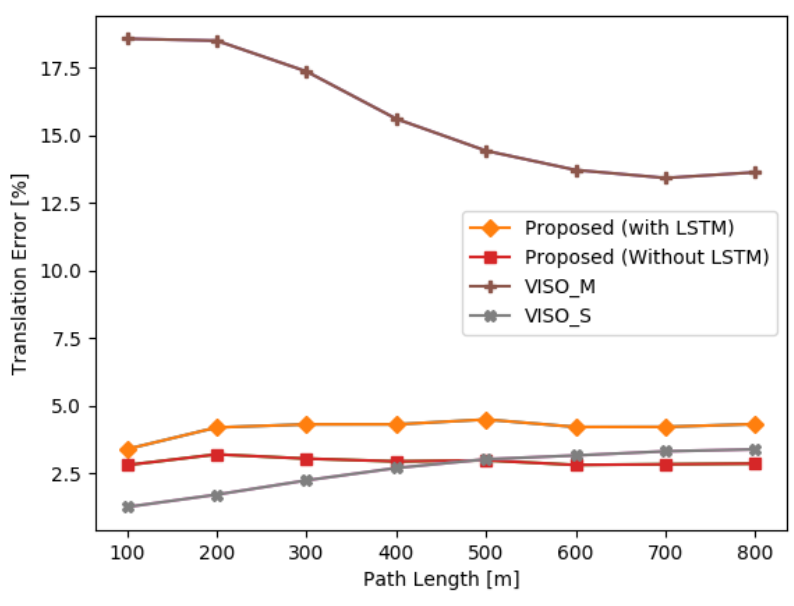

(a) Translation Error vs. Path Length

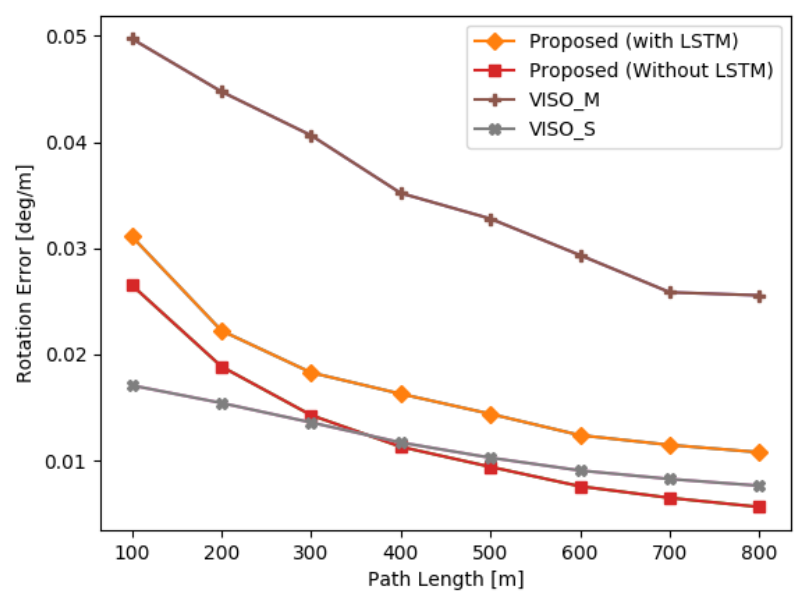

(c) Rotation Error vs. Path Length

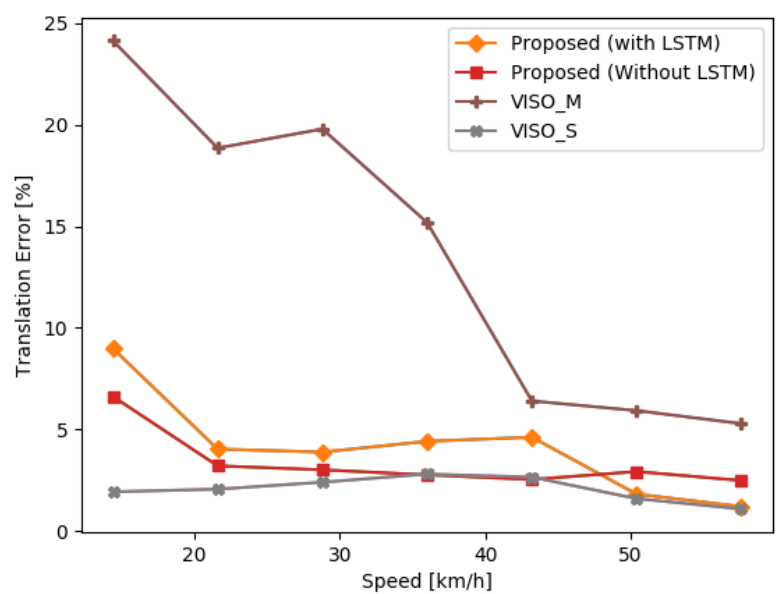

(b) Translation Error vs. Speed

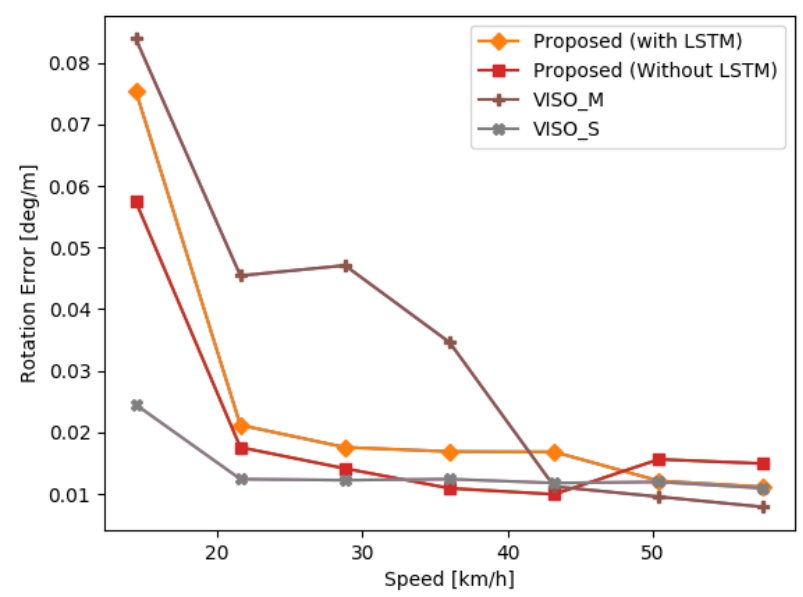

(d) Rotation Error vs. Speed

Figure 4. Comparison of the proposed method based on the KITTI evaluation kit. 


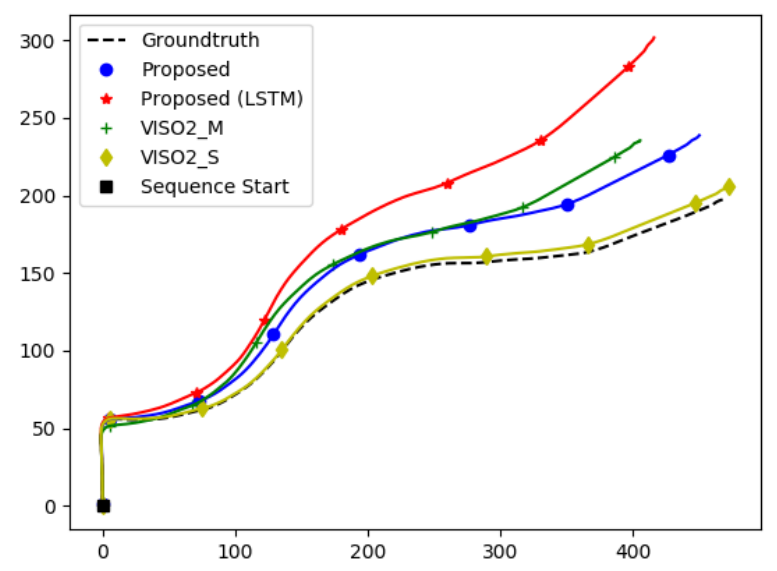

(a) Sequence 03

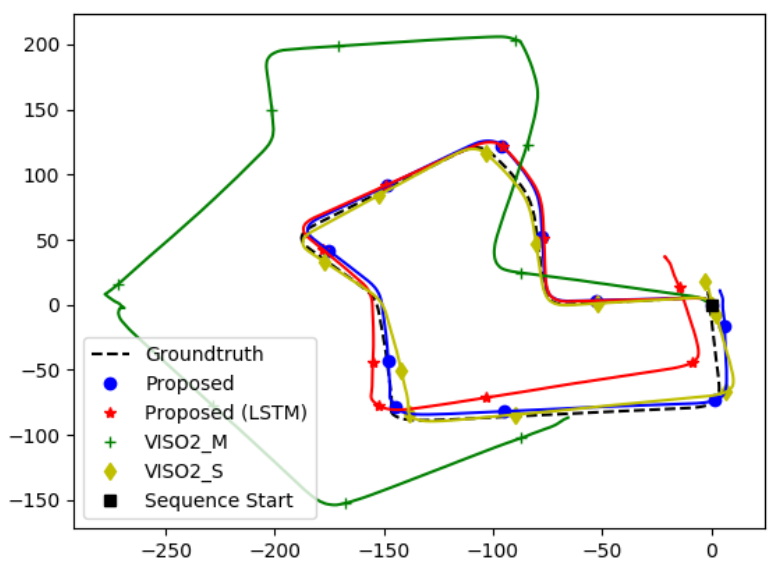

(c) Sequence 07

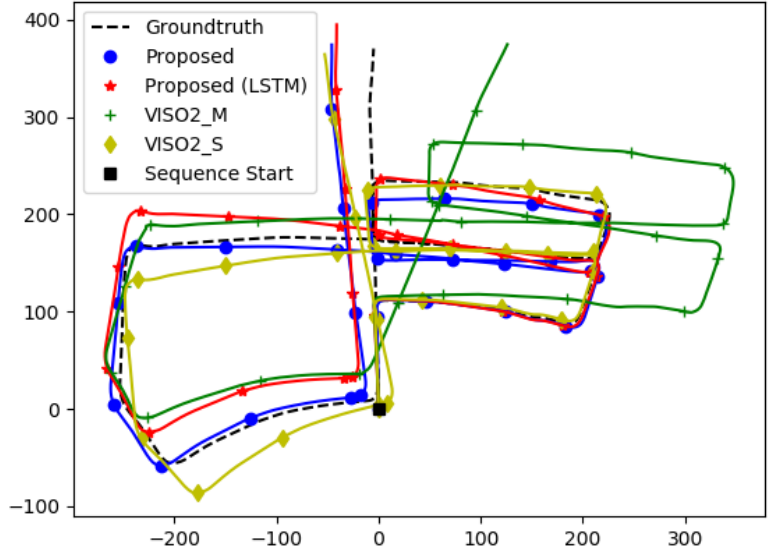

(b) Sequence 05

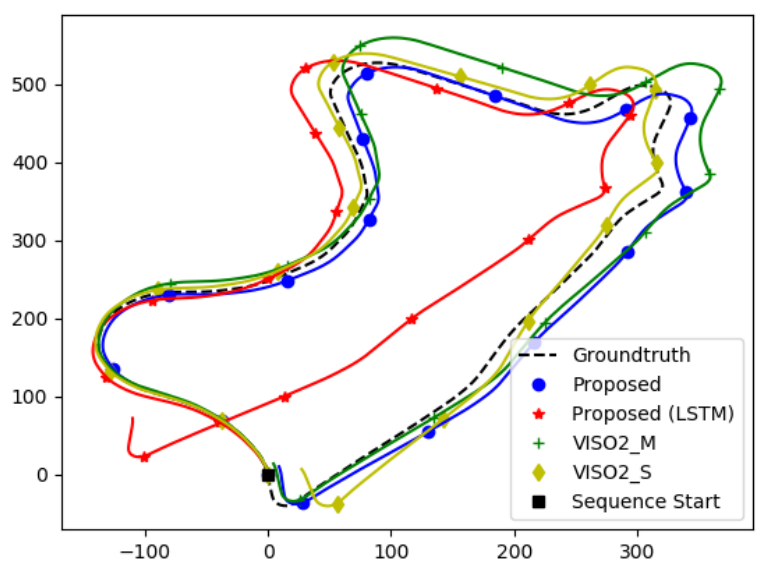

(d) Sequence 09

Figure 5. Comparison of our proposed method with VISO2.

\subsection{Analysis}

The averaged quantitative results are given in Table 2. We outperformed our monocular baselines (VISO2_M, MagicVO) on unknown environments and came close to the stereo-based approach (VISO_S). The stereo-based configuration uses multiple perspectives for better scene understanding and reduces the error caused by drift, while our approach uses deep learning to generate robust spatio-temporal feature descriptions, significantly outperforming traditional monocular methods.

The dataset consists of dynamic scenes with multiple moving objects, and was captured at $10 \mathrm{fps}$ with the car moving at high speeds. This results in large displacements between frames. It can prove challenging for traditional feature-based approaches to perform feature-matching between distant frames. A neural network might prove to be invariant to such faults, as the network takes many features into consideration.

Interestingly, LSTMs reduced our accuracy in a counter-intuitive manner. While the LSTMs managed to model the relationships between RGB image pairs, they fail to do so when working with optical flow images. This could be due to the prevalence of temporal feature representations, since RGB image pairs use the same image twice when iterating through a sequence, which is missing when working with optical flow since optical flow is scene-invariant. Subsequent optical flows can differ, whereas the RGB frame at time $t$ 
will be shared with both the frames at time $t-1$ and $t+1$. Figure $4 \mathrm{~b}$,d indicates that using LSTMs helped at high speeds when there are large displacements between frames, and it is able to use sequential information to output an improved estimate. There are also limited samples in the dataset with the car driving at speeds greater than 50 or slower than 20 . We believe more experimentation is required in assessing the role of LSTMs when working with optical flow feature representations.

Figure 5 also indicates the errors are not evenly distributed. While straight roads show little divergence, errors around turns and corners are high, which skews the global trajectory when a turn is encountered. Figure $4 b$,d shows errors increase significantly when the car is moving at a slow speed. Most of the samples consist of the car driving in a straight manner with uniform speed. The car occasionally slows down to turn or come to a stop. The lack of balanced data is a big drawback for deep learning against a stereo baseline.

With errors concentrated around turns, some frames contribute more to the relative errors which get accumulated over a trajectory. A loss function that can explicitly enforce the relationship between subsequent frames and weigh the contribution of each frame would be ideal. The averaged root-mean-square error is not sufficient where data are not weighted uniformly. Conventional methods perform feature extraction and evaluate whether a frame is viable for pose estimation and can contribute to the global trajectory. However, a neural network, when given an input, will always provide an output. Neural networks assume all inputs have an equal contribution. A biased network that can weight the input can get better results.

\section{Conclusions}

In this paper, we presented a deep learning-based solution for visual odometry (VO). We validated that CNNs are capable of predicting camera motion with only optical flow as input. Our proposed solution outperformed $\mathrm{MagicVO}$, which used features extracted from RGB images and modelled the VO pipeline using bi-directional LSTMs.

While our initial experiments surpassed VISO2_M and MagicVO (monocular baselines), the stereo-based approach still scored better. Additionally, augmenting our approach with LSTMs showed interesting behaviour that we would like to pursue in further studies. However, we believe that a bigger dataset would help in achieving better results.

For future work, we will test and validate our network on more datasets. We will also explore the architecture search space for a network with an induction bias more tuned for visual odometry.

Author Contributions: Conceptualization and Literature Review, T.P. and D.P.; Methodology and Experimentation, T.P.; Review, D.P. and J.B.; Supervision D.P., J.B., D.M. All authors have read and agreed to the published version of the manuscript.

Funding: This research received no external funding.

Institutional Review Board Statement: Not applicable.

Informed Consent Statement: Not applicable.

Data Availability Statement: The KITTI Dataset [40] used for this study can be accessed at http: / / www.cvlibs.net/datasets/kitti/ (accessed on 27 January 2021).

Conflicts of Interest: The authors declare no conflict of interest.

\section{References}

1. Scaramuzza, D.; Fraundorfer, F. Visual Odometry [Tutorial]. IEEE Robot. Autom. Mag. 2011, 18, 80-92. [CrossRef]

2. Yang, C.; Mark, M.; Larry, M. Visual odometry on the Mars Exploration Rovers. In Proceedings of the 2005 IEEE International Conference on Systems, Man and Cybernetics, Waikoloa, HI, USA, 12 October 2005; Volume 1, pp. 903-910. [CrossRef]

3. Corke, P.; Strelow, D.; Singh, S. Omnidirectional visual odometry for a planetary rover. In Proceedings of the 2004 IEEE/RSJ International Conference on Intelligent Robots and Systems (IROS) (IEEE Cat. No.04CH37566), Sendai, Japan, 28 September-2 October 2004; Volume 4, pp. 4007-4012. [CrossRef] 
4. Howard, A. Real-time stereo visual odometry for autonomous ground vehicles. In Proceedings of the 2008 IEEE/RSJ International Conference on Intelligent Robots and Systems, Nice, France, 22-26 September 2008; pp. 3946-3952. [CrossRef]

5. Wang, R.; Schworer, M.; Cremers, D. Stereo DSO: Large-Scale Direct Sparse Visual Odometry With Stereo Cameras. arXiv 2017, arXiv:1708.07878.

6. Huang, A.S.; Bachrach, A.; Henry, P.; Krainin, M.; Fox, D.; Roy, N. Visual odometry and mapping for autonomous flight using an RGB-D camera. In Robotics Research; Christensen, H., Khatib, O., Eds.; Springer Tracts in Advanced Robotics; Springer: Cham, Switzerland, 2011; Volume 100.

7. Kerl, C.; Sturm, J.; Cremers, D. Robust odometry estimation for RGB-D cameras. In Proceedings of the 2013 IEEE International Conference on Robotics and Automation, Karlsruhe, Germany, 6-10 May 2013; pp. 3748-3754. [CrossRef]

8. Leutenegger, S.; Lynen, S.; Bosse, M.; Siegwart, R.; Furgale, P. Keyframe-based visual-inertial odometry using nonlinear optimization. Int. J. Robot. Res. 2015, 34, 314-334. [CrossRef]

9. Bloesch, M.; Omari, S.; Hutter, M.; Siegwart, R. Robust visual inertial odometry using a direct EKF-based approach. In Proceedings of the 2015 IEEE/RSJ International Conference on Intelligent Robots and Systems (IROS), Hamburg, Germany, 28 September-2 October 2015; pp. 298-304. [CrossRef]

10. Yang, N.; Wang, R.; Gao, X.; Cremers, D. Challenges in Monocular Visual Odometry: Photometric Calibration, Motion Bias, and Rolling Shutter Effect. IEEE Robot. Autom. Lett. 2018, 3, 2878-2885. [CrossRef]

11. Nister, D.; Naroditsky, O.; Bergen, J. Visual odometry. In Proceedings of the 2004 IEEE Computer Society Conference on Computer Vision and Pattern Recognition, 2004. CVPR 2004, Washington, DC, USA, 27 June-2 July 2004; Volume 1, p. I. [CrossRef]

12. Mur-Artal, R.; Montiel, J.M.M.; Tardós, J.D. ORB-SLAM: A Versatile and Accurate Monocular SLAM System. arXiv 2015, arXiv:1502.00956.

13. Geiger, A.; Ziegler, J.; Stiller, C. StereoScan: Dense 3d reconstruction in real-time. In Proceedings of the 2011 IEEE Intelligent Vehicles Symposium (IV), Baden, Germany, 5-9 June 2011; pp. 963-968. [CrossRef]

14. Harris, C.; Stephens, M. A combined corner and edge detector. In Proceedings of the Alvey Vision Conference, Manchester, UK, 31 August-2 September 1988; pp. 147-151.

15. Engel, J.; Koltun, V.; Cremers, D. Direct Sparse Odometry. arXiv 2016, arXiv:1607.02565.

16. Howard, A.G.; Zhu, M.; Chen, B.; Kalenichenko, D.; Wang, W.; Weyand, T.; Andreetto, M.; Adam, H. MobileNets: Efficient Convolutional Neural Networks for Mobile Vision Applications. arXiv 2017, arXiv:1704.04861v1.

17. He, K.; Zhang, X.; Ren, S.; Sun, J. Deep Residual Learning for Image Recognition. arXiv 2015, arXiv:1512.03385v1.

18. Szegedy, C.; Liu, W.; Jia, Y.; Sermanet, P.; Reed, S.E.; Anguelov, D.; Erhan, D.; Vanhoucke, V.; Rabinovich, A. Going Deeper with Convolutions. arXiv 2014, arXiv:1409.4842.

19. Sabour, S.; Frosst, N.; Hinton, G.E. Dynamic Routing Between Capsules. arXiv 2017, arXiv:1710.09829.

20. Pan, X.; Shi, J.; Luo, P.; Wang, X.; Tang, X. Spatial As Deep: Spatial CNN for Traffic Scene Understanding. arXiv 2017, arXiv:1712.06080.

21. Fraundorfer, F.; Scaramuzza, D. Visual Odometry: Part II: Matching, Robustness, Optimization, and Applications. IEEE Robot. Autom. Mag. 2012, 19, 78-90. [CrossRef]

22. Forster, C.; Pizzoli, M.; Scaramuzza, D. SVO: Fast semi-direct monocular visual odometry. In Proceedings of the 2014 IEEE International Conference on Robotics and Automation (ICRA), Hong Kong, China, 31 May-7 June 2014; pp. 15-22. [CrossRef]

23. Engel, J.; Cremers, D. LSD-SLAM: Large-scale direct monocular SLAM. In Computer Vision-ECCV 2014; Lecture Notes in Computer Science; Fleet, D., Pajdla, T., Schiele, B., Tuytelaars, T., Eds.; Springer: Cham, Switzerland, $2014 ;$ Volume 8690.

24. Wang, S.; Clark, R.; Wen, H.; Trigoni, N. DeepVO: Towards End-to-End Visual Odometry with Deep Recurrent Convolutional Neural Networks. arXiv 2017, arXiv:1709.08429.

25. Jiao, J.; Jiao, J.; Mo, Y.; Liu, W.; Deng, Z. MagicVO: An End-to-End Hybrid CNN and Bi-LSTM Method for Monocular Visual Odometry. IEEE Access 2019, 7, 94118-94127. [CrossRef]

26. Muller, P.; Savakis, A. Flowdometry: An Optical Flow and Deep Learning Based Approach to Visual Odometry. In Proceedings of the 2017 IEEE Winter Conference on Applications of Computer Vision (WACV), Santa Rosa, CA, USA, 24-31 March 2017; pp. 624-631. [CrossRef]

27. Parisotto, E.; Chaplot, D.S.; Zhang, J.; Salakhutdinov, R. Global Pose Estimation with an Attention-based Recurrent Network. arXiv 2018, arXiv:1802.06857.

28. Fischer, P.; Dosovitskiy, A.; Ilg, E.; Häusser, P.; Hazirbas, C.; Golkov, V.; van der Smagt, P.; Cremers, D.; Brox, T. FlowNet: Learning Optical Flow with Convolutional Networks. arXiv 2015, arXiv:1504.06852.

29. Costante, G.; Mancini, M.; Valigi, P.; Ciarfuglia, T.A. Exploring Representation Learning With CNNs for Frame-to-Frame Ego-Motion Estimation. IEEE Robot. Autom. Lett. 2016, 1, 18-25. [CrossRef]

30. Costante, G.; Ciarfuglia, T.A. LS-VO: Learning Dense Optical Subspace for Robust Visual Odometry Estimation. arXiv 2017, arXiv:1709.06019.

31. Mur-Artal, R.; Tardós, J.D. ORB-SLAM2: An Open-Source SLAM System for Monocular, Stereo and RGB-D Cameras. arXiv 2016, arXiv:1610.06475.

32. Sener, O.; Koltun, V. Multi-Task Learning as Multi-Objective Optimization. arXiv 2018, arXiv:1810.04650v2. 
33. Jiang, H.; Sun, D.; Jampani, V.; Yang, M.; Learned-Miller, E.G.; Kautz, J. Super SloMo: High Quality Estimation of Multiple Intermediate Frames for Video Interpolation. arXiv 2018, arXiv:1712.00080.

34. DLSS 2.0-Image Reconstruction for Real-Time Rendering with Deep Learning. Available online: https://www.nvidia.com/enus/on-demand/session/gtcsj20-s22698/ (accessed on 27 January 2021).

35. Barron, J.L.; Fleet, D.J.; Beauchemin, S.S. Performance of Optical Flow Techniques. Int. J. Comput. Vision 1994, $12,43-77$. [CrossRef]

36. Ilg, E.; Mayer, N.; Saikia, T.; Keuper, M.; Dosovitskiy, A.; Brox, T. FlowNet 2.0: Evolution of Optical Flow Estimation with Deep Networks. arXiv 2016, arXiv:1612.01925.

37. Hui, T.; Tang, X.; Loy, C.C. LiteFlowNet: A Lightweight Convolutional Neural Network for Optical Flow Estimation. arXiv 2018, arXiv:1805.07036.

38. Sun, D.; Yang, X.; Liu, M.; Kautz, J. PWC-Net: CNNs for Optical Flow Using Pyramid, Warping, and Cost Volume. arXiv 2017, arXiv:1709.02371.

39. Geiger, A.; Lenz, P.; Urtasun, R. Are we ready for Autonomous Driving? The KITTI Vision Benchmark Suite. In Proceedings of the Conference on Computer Vision and Pattern Recognition (CVPR), Providence, RI, USA, 16-21 June 2012.

40. Geiger, A.; Lenz, P.; Stiller, C.; Urtasun, R. Vision meets Robotics: The KITTI Dataset. Int. J. Robot. Res. (IJRR) 2013. [CrossRef]

41. O'Malley, T.; Bursztein, E.; Long, J.; Chollet, F.; Jin, H.; Invernizzi, L. Keras Tuner. Available online: https:/ / github.com/kerasteam/keras-tuner (accessed on 5 August 2020).

42. Graves, A.; Mohamed, A.; Hinton, G.E. Speech Recognition with Deep Recurrent Neural Networks. arXiv 2013, arXiv:1303.5778.

43. Lipton, Z.C. A Critical Review of Recurrent Neural Networks for Sequence Learning. arXiv 2015, arXiv:1506.00019. 